\title{
Literature Update on Melanocytic Nevi and Pigmented Lesions in the Pediatric Population
}

\author{
Jillian F. Rork • Elena B. Hawryluk • Marilyn G. Liang
}

Published online: 12 September 2012

(C) Springer Science+Business Media, LLC 2012

\begin{abstract}
Pigmented lesions and melanocytic nevi are commonly evaluated lesions in pediatric dermatology clinics and the rising incidence of melanoma has increased public awareness of malignant potential. Diagnosis and management of pigmented lesions, especially dysplastic nevi, medium and large congenital melanocytic nevi (CMN), and Spitz nevi, can be particularly challenging. We summarize the recent literature for melanocytic nevi and pigmented lesions as relevant to the practice of pediatric dermatology with particular attention to dermatoscopic techniques, histopathologic interpretation, molecular biology, and management recommendations for CMN and Spitz nevi.
\end{abstract}

Keywords Nevus $\cdot$ Congenital melanocytic nevus $\cdot$ Spitz nevus $\cdot$ Melanoma

\section{Introduction}

Pigmented lesions are commonly evaluated lesions in pediatric dermatology clinics. These include congenital melanocytic nevi (CMN) and acquired melanocytic nevi (AMN), dysplastic nevi, Spitz nevi, Becker nevi, halo nevi, blue nevi, and nevus spilus. While the vast majority of nevi in

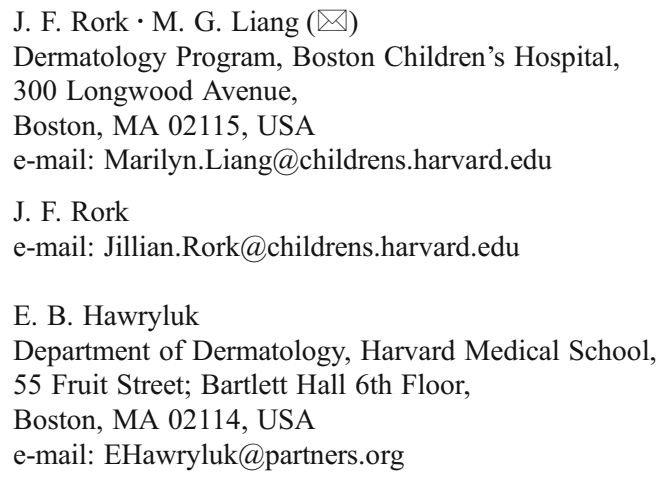

the pediatric population are benign, the rising incidence of melanoma among the general population has increased the awareness of pigmented lesions and concern for malignant potential. Fortunately, melanoma in the pediatric population is rare, accounting for 1-3\% of childhood malignancies and representing approximately $2 \%$ of all melanoma diagnoses $[1,2]$. Despite this rarity, the course of melanoma in children has a similar prognosis as adults, and therefore clinicians should assess all nevi for atypical features [3].

In this review article, we summarize the recent literature and advances in our understanding of melanocytic nevi and pigmented lesions as relevant to the practice of pediatric dermatology. We have focused on articles published after 2010 to provide the most up-to-date perspective for readers. Whereas the discussion of pigmented lesions traditionally includes melanoma, we will not provide an explicit section on this topic given the extensive amount of recently published melanoma literature. Instead, attention will be placed on advances in dermatoscopic techniques, histopathologic interpretation, molecular biology, and management recommendations, especially for CMN and Spitz nevi.

\section{Congenital Melanocytic Nevi}

CMN are strictly regarded as present at the time of birth, although children can develop pigmented lesions with the clinical and histological characteristics of $\mathrm{CMN}$ during the first year of life (Fig. 1a) [4]. CMN are commonly classified according to their greatest diameter in adulthood: small $(<1.5 \mathrm{~cm})$, medium $(1.5-19.9 \mathrm{~cm})$, and large or giant $(>20 \mathrm{~cm})$, although some reserve the term "giant" for nevi with diameters greater than $50 \mathrm{~cm}$. While patients with large and giant $\mathrm{CMN}$ have the greatest overall lifetime risk of melanoma, estimated between $4.5 \%$ and $10 \%$ [5], all congenital nevi should be considered as potential precursor 

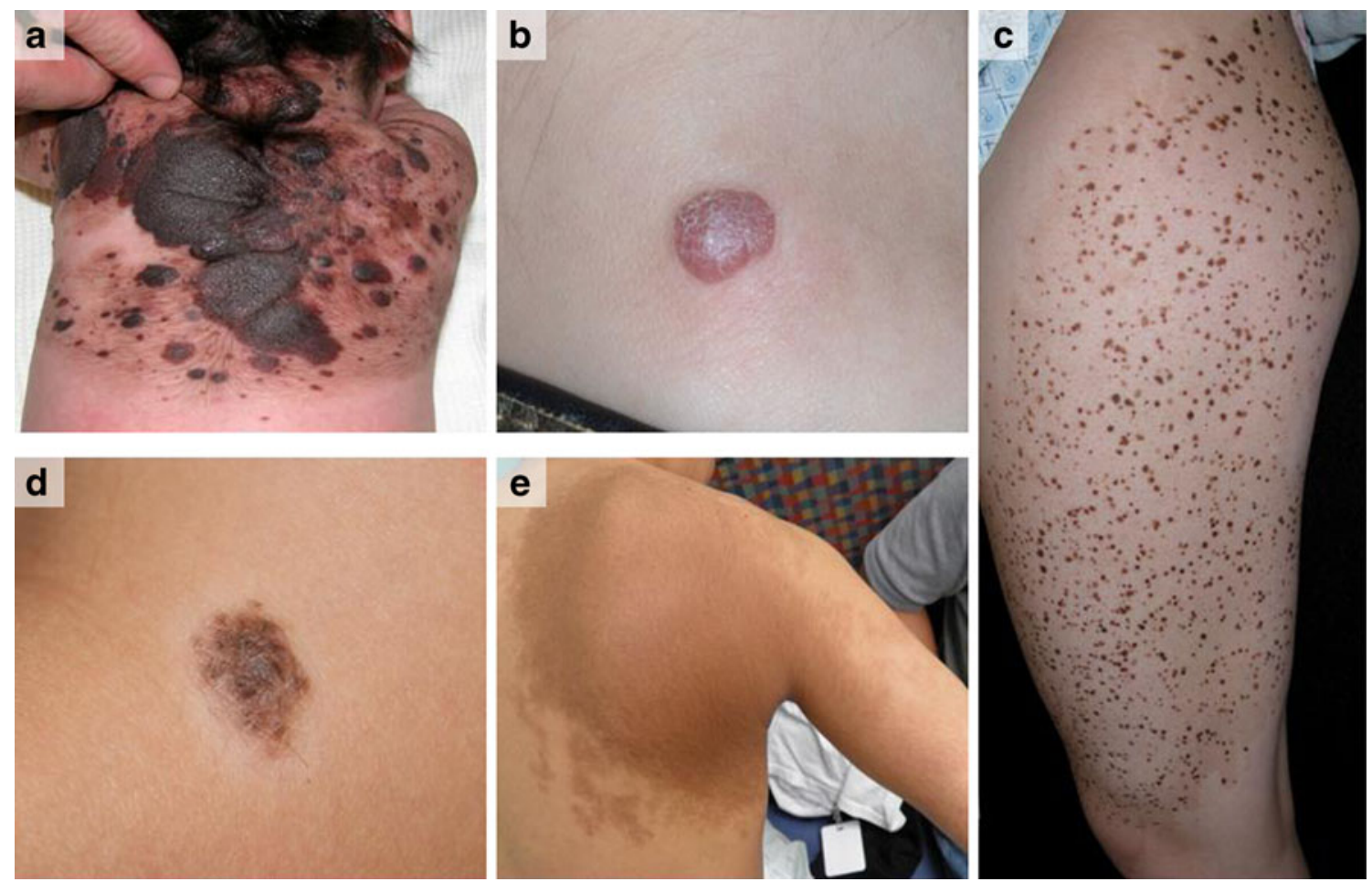

ankle. c 14-year-old female with a nevus spilus on the left thigh and

Fig. 1 Pigmented lesions. a 2-month-old female with a giant congenital melanocytic nevus (CMN) involving posterior scalp, shoulders, and back. b 4-year-old male with a Spitz nevus on the right lateral buttock. d 7-year-old male with a halo nevus on the right lateral knee. e 15 -year-old male with a Becker nevus over the right scapula lesions and patients and parents should be educated to monitor for changes in appearance.

Dermatoscopic features have been proposed as a potential tool to better understand the natural history of $\mathrm{CMN}$ and how they differ from acquired nevi. Stinco and colleagues recently published a nonrandomized observational study of 133 nevi in 2-year-old children to identify dermatoscopic differences between melanocytic lesions [6•]. Results showed no statistical significance between the dermatoscopic features of CMN present at birth and nevi present during the first 2 years of life. The predominant patterns of all melanocytic nevi were globular (50\%), consistent with prior studies [7]. They also observed a reticular pattern among nevi present at birth (28\%), which contradicts previous descriptions of a predominately reticular pattern in CMN for individuals 12 years or older $[7,8]$. The presence of both globular and reticular patterns in CMN raises questions regarding nevus evolution and pathways responsible for nevogenesis.

Minigawa and colleagues also recently published on the dermatoscopic characteristics of CMN affecting acral volar skin [9•]. A combination of crista dotted and parallel furrow patterns, termed "peas-in-a-pod" pattern, was the most common dermatoscopic feature noted in the acral CMN (38 \%). In contrast, most cases of acral AMN exhibit the parallel furrow, latticelike, or fibrillar pattern, and the majority of early acral melanomas demonstrate a parallel ridge pattern
$[10,11]$. It should be mentioned that one acral CMN in this study showed the parallel ridge pattern similar to acral melanomas; however, the lesion was clinically stable for several years, suggestive of a benign nevus. Interestingly, another potential confounding diagnosis among the pediatric population with the concerning parallel ridge pattern is termed "playstation purpura" [12]. These asymptomatic pigmented macules on acral volar skin are caused by repetitive trauma from videogame controllers and present with dermatoscopic features of homogeneous reddish-brown pigment in a parallel ridge pattern. In conclusion, dermatoscopic findings may help differentiate acral CMN from both acral AMN and melanoma which is particularly relevant for the non-white population for whom acral volar skin is the most prevalent site of melanoma.

The low incidence of large and giant CMN poses a challenge in studying melanoma risk and clinical outcomes among these patients. Whereas approximately $1 \%$ of newborns have a small CMN, giant CMN are estimated to occur in 1 in 500,000 newborns $[13,14]$. A recent nationwide retrospective chart review in Korea specifically addressed the risk of melanoma in giant $\mathrm{CMN}$ in the Asian population [15]. Among 131 patients enrolled in the study, two cutaneous and one meningeal melanoma were reported $(2.3 \%)$. Patients who developed melanoma were ages 6 years, 14 years, and 70 years. Although most giant CMN melanomas are considered to develop within 
the first 10 years of life [16], this wide age range emphasizes the importance of lifelong follow-up.

An important consideration for patients with large CMN, particularly those located over the lower spine with satellite nevi, is evaluation for neurocutaneous melanosis (NCM). NCM is a rare disorder characterized by a proliferation of benign or malignant nevus cells in the central nervous system (CNS). Patients with NCM are more prone to develop melanoma of the CNS, epilepsy, and other neurological symptoms related to spinal cord compression and increased intracranial pressure. Although recent literature includes case reports and general reviews [17-19], no advances have been made in NCM management, and there are no guidelines for workup or surveillance. In our clinical practice, a baseline brain MRI is offered to patients with large CMN overlying the lower spine which may prompt referral to neurology or ongoing monitoring, although some parents choose to forego imaging given the limited available interventions.

Another difficult aspect of CMN management is the histopathologic interpretation of melanoma within CMN. In particular, proliferative nodules $(\mathrm{PN})$ arising within CMN pose significant diagnostic challenges. PN can exhibit an atypical histologic appearance that is difficult to distinguish from melanoma. In an attempt to improve histopathologic interpretation, a recent article was published on the histologic, immunohistochemistry (IHC), and molecular analysis of atypical $\mathrm{PN}\left[20^{\bullet}\right.$. In summary, histologic features of atypical PN, such as sharp demarcation, expansile growth, epidermal effacement, nuclear pleomorphism, and increased mitoses, were found to differ significantly from those of benign PN. IHC levels of Ki-67 and PHH3 also were significantly higher in atypical PN. Because the immunohistochemical profile of atypical PN is not significantly different from melanoma, the study authors recommended treating atypical PN conservatively as borderline melanocytic lesions requiring complete excision with adequate margins and regular clinical follow-up.

We find the clinical and histological assessment of PN particularly challenging. In our clinic, PN with concerning clinical characteristics, such as rapid growth, ulceration, or change in color, merit histopathologic evaluation. Regarding management of atypical PN, we recommend biopsy of the tissue of concern for diagnostic purpose, with clear designation on the pathology requisition that the sample of interest is arising within a CMN. Subsequent management is guided by pathology. We follow CMN patients annually, with the understanding that any concerning clinical changes require prompt evaluation.

During the past decade, laser treatment of CMN has become a particular area of interest. A variety of lasers have been attempted, including pigment-specific lasers, resurfacing lasers, and more currently, a combination of both. August and colleagues recently published an article on the cosmetic outcomes and side effects of carbon dioxide and pigment-specific lasers in the treatment of medium-sized CMN [21]. Funayama and colleagues also treated large-togiant CMN with combination pulse-dye laser and Q-switched ruby laser [22]. Whereas the study authors posed several encouraging conclusions, the results have not changed our clinical practice. The current medical literature lacks critical assessment on the potential long-term dysplastic effects of laser on $\mathrm{CMN}$, including masking and/or potentially precipitating melanoma. Until further evidence is published, we limit performing laser treatment of CMN.

\section{Acquired Melanocytic Nevi}

The annual transformation rate of any single melanocytic nevus into melanoma is exceedingly low, estimated at $0.0005 \%$ or less for patients younger than age 40 years [23]; nonetheless, one risk factor for melanoma is a high number of AMN [24]. Children tend to have few AMN, but these lesions increase in number with age until mid-life [25]. Similar to CMN as discussed above, dermatoscopic studies suggest AMN patterns also are age-dependent. Zalaudek and colleagues published a cross-sectional study attempting to subclassify AMN by dermatoscopic patterns and anatomical location [26•]. Results were similar to previous observations, which showed that globular-pattern nevi were the most prevalent AMN on the upper trunk in children/adolescents and a reticular pattern was the most common AMN pattern after the second decade of life on the shoulders and middle back [27, 28]. Although controversy exists regarding the most prevalent dermatoscopic pattern of pediatric nevi, Zalaudek and colleagues' findings support a hypothesis that globular versus reticular nevi represent distinct pathways of nevogenesis. The authors suggest an early onset of globular nevi, as opposed to the later predominance of reticular nevi, supports the theory that globular nevi are more likely to be congenitally determined and reticular nevi may be driven by exogenous influences.

As suggested, several factors may contribute to the development of $\mathrm{AMN}$, including genetic predisposition, skin type, and environmental factors, such as ultraviolent (UV) light. To further categorize the effect of UV radiation in children, Mahé and colleagues performed skin examinations during a 2-year period on 660 11-year-old children [29]. As predicted, the AMN count was found to be higher in children who practiced outdoor sports. These results suggest that sun-protection campaigns should be aimed particularly at children who participate in outdoor athletics. In our practice, we recommend that all patients practice conservative sun protection, including repeated application of sunscreen when outdoors for prolonged durations.

Researchers continue to investigate potential molecular events necessary for the development of melanoma from 
melanocytic nevi. Previous literature suggests AMN often harbor mutations overlapping with those of primary and metastatic melanoma. For example, BRAF mutations were identified in $82 \%$ of nevi; therefore, BRAF mutation alone is thought to be insufficient for the development of melanoma [30]. In fact, melanocytic nevi can be relatively stable for decades despite the presence of activating BRAF mutations. In contrast, ras mutations were not identified among normal or dysplastic nevi [31], although another study found NRAS mutations in $18.2 \%$ of common AMN [32]. We look forward to clarifying studies and a future role for molecular genetics in determining whether an AMN has the potential to transform into melanoma.

\section{Dysplastic Nevi}

First described in melanoma-prone families with a high prevalence of nevi [33-35], the term "dysplastic nevi" continues to be controversial. As summarized in a recent review in the Journal of the American Academy of Dermatology, uncertainty still remains whether dysplastic nevi represent a premalignant lesion $[36 \bullet \bullet, 37 \bullet \bullet]$. In the pediatric population, the frequency of histologically confirmed dysplastic nevi is reportedly extremely low [38]. Children who have a family history of melanoma appear to have a higher incidence of dysplastic moles [39], although the majority of patients with dysplastic nevi have no obvious familial pattern. One exception is familial atypical multiple mole-melanoma (FAMMM) syndrome, a disorder of autosomal dominant inheritance; the $C D K N 2 A$ gene, encoding a tumor suppressor gene p16, was found to be mutated in some patients with FAMMM in addition to other cancer types [40].

Nevi with concerning histopathological features are common when sampled from "special sites," such as the scalp or genitalia. Whereas melanoma of the scalp does occur in childhood [41], indiscriminate removal of scalp nevi may lead to heightened anxiety among patients and unnecessary procedures. To improve clinical management, Tcheung and colleagues recently sought to establish the typical clinical and dermatoscopic patterns of scalp nevi in children younger than age 18 years [42•]. Overall, older subjects and boys were found to harbor a larger proportion of scalp nevi. The most common dermatoscopic patterns were globular (57\%) and complex/reticular-globular (27 \%). Perifollicular hypopigmentation, with resultant clinically scalloped borders or variegation in pigmentation, also was a hallmark feature of scalp nevi. Dermatoscopy may therefore be a helpful diagnostic tool before considering excision of a scalp nevus.

Histological analysis of dysplastic nevi has limitations, and molecular studies are being investigated as a potential tool to classify nevi when a morphological distinction is challenging. Some dysplastic nevi have been found to exhibit unique gene expression patterns, mutation or altered expression of p16 and p53, and increased microsatellite instability $[37 \bullet \bullet]$. Accordingly, Husain and colleagues recently compared topographic profiles of cell cycle and kinetic regulators among common, low-grade atypical and high-grade atypical melanocytic nevi and found that highgrade atypical nevi accumulated microsatellite abnormalities [39]. Molecular analysis also may provide a more reliable predictor of future biological behavior of lesions. Whereas no current markers have been shown to predict behavior, Moore and colleagues recently demonstrated that a four-probe fluorescent in situ hybridization (FISH) assay correctly identified $83.8 \%$ of melanomas and additionally detected genetic abnormalities in $6.3 \%, 6.7 \%$, and $10.3 \%$ of nevi with mild, moderate, and severe histopathologic atypia, respectively [43]. These results suggest a potential correlation between the severity of dysplasia and genetic abnormalities detectable by FISH.

Several novel approaches have recently been attempted to discriminate between benign and malignant melanocytic lesions, including reflectance confocal microscopy [44] and RASSF10 promoter hypermethylation status [45]. Reflectance confocal microscopy is a noninvasive tool that provides instant visualization of skin structures at a quasihistopathologic resolution. Longo and colleagues report this technique may be able to identify differences in cell morphology and discern melanoma in the setting of preexisting nevi [46]. A separate study reported the utility of assessing for the status of a member of the Ras association domain family (RASSF), specifically RASSF10. The RASSF family consists of several tumor suppressor genes frequently silenced in human cancers. In contrast to benign melanocytic lesions, RASSF10 was found to be frequently hypermethylated in melanoma [45]. Further research is required for the clinical application of these techniques.

\section{Spitz Nevi}

Among pigmented lesions, a particularly exciting area of research focuses on Spitz nevi (Fig. 1b). Typically appearing in school-age children, a Spitz nevus, or spindle and epithelioid cell nevus, is composed of melanocytes with irregular nuclear shapes and abundant cytoplasm. These features can raise histopathologic concern for melanoma and, over the years, have created complex classification schemes. In addition to classic Spitz nevi, which are recognized as benign proliferations, certain Spitz tumors demonstrate extensive pleomorphic features indistinguishable from melanoma and are termed Spitzoid melanoma. There also is a cohort of so-called "Spitzoid tumors of uncertain malignant potential" or "STUMP" lesions whose name reflects the challenge in diagnosis [47]. For simplicity, we will use the general 
term "Spitz tumor" to describe the range of lesions with Spitzoid features.

A two-part comprehensive literature review of Spitz tumors by Luo and colleagues reviews the topic of Spitz nevi broadly and highlights several potential histopathologic features more commonly associated with metastatic behavior: ulceration, significant Breslow thickness, number of mitotic figures, and deep/atypical mitotic figures [48••]. Regarding Spitzoid melanoma diagnosis, a recent description of five new histopathologic subtypes of Spitzoid melanoma-genuine, uniform, packed, polypoid, and pigmented - aims to provide useful parameters to improve diagnostic confidence [49].

Despite these insights, certain histopathologic observations within Spitz tumors continue to cloud diagnosis. One histopathologic feature of melanoma is "transepidermal melanocytic migration," which, in conjunction with cytologic atypia, is an important criterion for malignancy and also has been observed among benign melanocytic tumors, such as some Spitz tumors [50]. Given this histopathologic atypia and the dire clinical consequences of melanoma, the presence of these features in Spitz tumors can result in overdiagnosis of malignancy and unnecessary aggressive management.

Whereas the histopathologic diagnosis of Spitz tumors remains challenging, several newer diagnostic technologies are being employed. Luo and colleagues highlight many advances in molecular analysis and Spitz tumors, such as amplifications in chromosome 11p unique to a subset of Spitz tumors and a potentially distinct HRAS mutation profile in Spitz nevi [48••]. In January 2012, Gammon and colleagues reported the 9p21 FISH probe was a helpful adjunctive target in lesions with Spitzoid morphology [51]. Another study utilizing FISH and multiple ligation-dependent probe amplification (MLPA) for the 9p21 deletion also found alterations at 9p21 occurred more frequently in Spitz tumors versus Spitz nevi [52]. However, other recent studies have challenged the utility of molecular analysis for Spitz tumors. Martin and colleagues recommend cautious interpretation of FISH analysis given findings of similar cytogenetic alterations in Spitz tumors and melanoma [53].

Overall, attempts to use mutation analysis to distinguish Spitz tumors from melanoma have produced inconsistent results. A single diagnostic technique cannot be relied upon to provide proper diagnosis but could potentially be helpful when combined with other modalities. To support this perspective, Nardone and colleagues found a combination of clinical/dermatoscopic findings and FISH enhanced detection in a subset of patients with early melanoma, including cases where Spitz nevus was on the differential [54].

Immunohistochemistry also has been investigated as a means of differentiating Spitz tumors. Al Dhaybi and colleagues proposed the expression of p16 as a potential marker of childhood nodular Spitzoid malignant melanomas, which may aid in distinguishing this subtype from Spitz nevi [55]. Other recent diagnostic approaches include imaging mass spectrometry, which was able to classify Spitz nevi correctly with $97 \%$ sensitivity and $90 \%$ specificity based on proteomic differences [56]. In a small pilot study, hyperspectral data also was utilized to create an index at a molecular pigmentary level to diagnose melanoma, with a sensitivity of $90 \%$ and specificity of $84 \%$ [57].

Despite advances in our molecular understanding, management challenges remain. A study of long-term outcome of Spitz-type melanocytic tumors during a 15 -year period found that of 157 patients with Spitz-type melanocytic lesions, atypical Spitz tumors were associated with an increased melanoma risk, minimal lethal potential, and moderate risk of metastasis to the regional lymph node basin [58•]. The true malignant potential of these lesions is debated and a number of studies have examined the role for sentinel lymph node biopsy, demonstrating an overall positive rate of approximately $38 \%[48 \bullet$, $59 \bullet \cdot$. Sentinel lymph node biopsy is more likely to show micrometastasis of atypical Spitz tumors that are $>1 \mathrm{~cm}$ in diameter and ulcerated with subcutaneous involvement and mitoses $[48 \cdot \bullet, 59 \bullet \cdot]$. The biological significance of sentinel lymph node positivity is questionable given the disparity of outcomes. In contrast to melanoma, a Spitz nevus with positive nodal findings has a generally benign clinical course; however, a positive sentinel lymph node study often prompts complete lymphadenectomy and a potential year-long interferon course for these patients, causing both acute and long-term morbidity.

To summarize, common Spitz nevi may be monitored clinically or excised. Those with clinically unusual, changing, or concerning features are biopsied. Histopathologic diagnosis of an atypical Spitz tumor will prompt consideration and discussion of sentinel node biopsy, with a clear discussion with the parents regarding the high positive rate of sentinel node studies among Spitz tumors and the challenges in interpreting these results. For this reason, many pediatric patients forego sentinel lymph node study and after complete excision are followed clinically. Excision should be directed with a goal of complete removal of Spitz nevi, because subsequent recurrences of common Spitz nevi can present greater diagnostic challenges. For atypical Spitz tumors, some advocate for excision with wide margins per melanoma guidelines [60]. Spitz tumors in the adult population are managed more definitively, with complete excision and care more closely aligned with melanoma management, given the contrast in prognosis between pediatric and adult patients with Spitz tumors.

\section{Other Pigmented Lesions}

Becker nevi are characterized by macular hyperpigmentation with hypertrichosis and often occur on the upper trunk (Fig. 1e). Becker nevi are occasionally congenital but more 
commonly appear during adolescence due to a localized increase in androgen receptor sensitivity within the nevus [61]. A recent retrospective study of 118 children in Italy with a diagnosed Becker nevus showed a nearly equal distribution among sexes (47\% male) in contrast to the common assumption of predominance in adolescent males [62]. Hypertrichosis also was present in only one-third of cases as compared to the presence of terminal hairs in the Becker nevi of $70 \%$ of young Italian men [63]. The authors attribute this observation to the young age of the study patients, many of whom were preadolescent and thus had not experienced prominent hormone changes. The lack of hypertrichosis can make the diagnosis more challenging, especially in preadolescent patients.

Halo nevi generally present as a central pigmented lesion surrounded by a halo of hypo- or depigmentation (Fig. 1d). The central pigmented lesion is most commonly an AMN, but a halo also can occur around a CMN, blue nevus, Spitz nevus, and melanoma. The loss of pigmentation appears to be related to immunological destruction of melanocytes and nevus cells [64]. This theory is further supported by the fact that halo nevi are associated with vitiligo. A study of the prognostic value and clinical significance of halo nevi and vitiligo found the age of vitiligo onset among patients with halo nevi was significantly lower than patients without halo nevi $(P<$ 0.001 ) [65 ${ }^{\circ}$. In addition, a recent retrospective observational study of 125 patients found patients with multiple halo nevi to have a higher risk of vitiligo and other autoimmune diseases than patients with a single halo nevus [66].

The appearance of a halo is believed to correlate with the onset of nevus regression and eventual disappearance of the nevus. The amount of observed change can be concerning for patients and parents, and many present to the clinic fearing melanoma. Halo nevi can occur around melanomas, thought to signify the host immune system's recognition of the melanoma, and therefore the identification of a halo nevus should prompt complete cutaneous examination for suspicious pigmented lesions. This finding is believed to be rare in children; in one survey of 78 pediatric dermatologists, no diagnosis of melanoma was reported [67]. In an attempt to understand the natural history and timing of pigment changes in halo nevi, Aouthmany and colleagues conducted a retrospective chart review of 52 patients with 80 halo nevi [68 ${ }^{\bullet}$. Results demonstrated that a halo nevus typically persists for a decade or longer. They may progress through multiple stages of involution with an eventual return to normal-appearing skin; however, even these lesions persisted for an average of 7.8 years. Knowledge of the prolonged natural history of halo nevi may reassure patients and clinicians and avoid unnecessary surgical excision.

A blue nevus is comprised of arrested dendritic melanocytes within the dermis and the deep location results in a bluish-black clinical appearance due to the Tyndall effect of light scattering. Blue nevi, Mongolian spots, and nevi of Ota are all types of dermal melanocytosis, representing variants of the same physiological process. A recent case report of a young woman born with generalized congenital dermal melanocytosis who subsequently developed bilateral nevus of Ota and eruptive blue nevi highlights this association [69].

Nevus spilus is a brown patch that comprises scattered hyperpigmented macules or papules (Fig. 1c). Nevus spilus can present as a congenital or acquired nevoid disorder with the initial presentation often similar to a café-au-lait patch and subsequent development of superimposed pigmented nevi. Overlying hypertrichosis has been occasionally reported, and a small case series describes coarse hairs mainly arising from the background pigmented area [70]. The development of melanoma is thought to be rare, although a recent case report was published of a 64-year-old woman who developed nodular achromic melanoma within a nevus spilus [71]. We recommend longitudinal serial examinations and excision of atypical lesions or areas of clinical change.

\section{Conclusions}

While the prevalence of melanoma in the pediatric population is exceedingly low, a high index of suspicion should be maintained for any rapidly growing or otherwise changing pigmented skin lesion, and excisional biopsy should be pursued for complete histopathologic evaluation. With further advances in dermatoscopy and the molecular biology of pigmented lesions, we will have increasing confidence in the diagnosis and management of benign and malignant pigmented lesions.

Acknowledgments The authors acknowledge Robert Sidbury, $\mathrm{MD}$, and Jennifer T. Huang, MD, for contributing photographs to this article.

Disclosures The authors reported no conflicts of interest relevant to this article.

\section{References}

Papers of particular interest, published recently, have been highlighted as:

- Of importance

-. Of major importance

1. Young JL Jr, Percy CL, Asire AJ, et al. Cancer incidence and mortality in the United States, 1973-77. Natl Cancer Inst Monogr. 1981:1-187.

2. Bader JL, Li FP, Olmstead PM, et al. Childhood malignant melanoma. Incidence and etiology. Am J Pediatr Hematol Oncol. 1985;7:341-5. 
3. Schmid-Wendtner MH, Berking C, Baumert J, et al. Cutaneous melanoma in childhood and adolescence: an analysis of 36 patients. J Am Acad Dermatol. 2002;46:874-9.

4. Clemmensen OJ, Kroon S. The histology of "congenital features" in early acquired melanocytic nevi. J Am Acad Dermatol. 1988; 19:742-6.

5. Lyon VB. Congenital melanocytic nevi. Pediatr Clin North Am. 2010;57:1155-76.

6. - Stinco G, Argenziano G, Favot F, et al. Absence of clinical and dermoscopic differences between congenital and noncongenital melanocytic naevi in a cohort of 2-year-old children. Br J Dermatol. 2011;165:1303-7. This study showed no statistical significance between the dermatoscopic features of CMN present at birth and nevi presenting during the first 2 years of life.

7. Seidenari S, Pellacani G, Martella A, et al. Instrument-, age- and sitedependent variations of dermoscopic patterns of congenital melanocytic naevi: a multicentre study. Br J Dermatol. 2006;155:56-61.

8. Changchien L, Dusza SW, Agero AL, et al. Age- and site-specific variation in the dermoscopic patterns of congenital melanocytic nevi: an aid to accurate classification and assessment of melanocytic nevi. Arch Dermatol. 2007;143:1007-14.

9. - Minagawa A, Koga H, Saida T. Dermoscopic characteristics of congenital melanocytic nevi affecting acral volar skin. Arch Dermatol. 2011;147:809-13. This study showed that a combination of crista dotted and parallel furrow patterns, termed "peas-in-apod" pattern, was the most common dermatoscopic feature noted in acral CMN.

10. Oguchi S, Saida T, Koganehira Y, et al. Characteristic epiluminescent microscopic features of early malignant melanoma on glabrous skin. A videomicroscopic analysis. Arch Dermatol. 1998;134:563-8.

11. Saida T, Oguchi S, Miyazaki A. Dermoscopy for acral pigmented skin lesions. Clin Dermatol. 2002;20:279-85.

12. Robertson SJ, Leonard J, Chamberlain AJ. PlayStation purpura. Australas J Dermatol. 2010;51:220-2.

13. Karvonen SL, Vaajalahti P, Marenk M, et al. Birthmarks in 4346 Finnish newborns. Acta Derm Venereol. 1992;72:55-7.

14. Castilla EE, da Graca Dutra M, Orioli-Parreiras IM. Epidemiology of congenital pigmented naevi: I. Incidence rates and relative frequencies. Br J Dermatol. 1981;104:307-15.

15. Yun SJ, Kwon OS, Han JH, et al. Clinical characteristics and risk of melanoma development from giant congenital melanocytic naevi in Korea: a nationwide retrospective study. Br J Dermatol. 2012;166:115-23.

16. Marghoob AA, Agero AL, Benvenuto-Andrade C, Dusza SW. Large congenital melanocytic nevi, risk of cutaneous melanoma, and prophylactic surgery. J Am Acad Dermatol. 2006;54:868-70.

17. Agero AL, Busam KJ, Benvenuto-Andrade C, et al. Reflectance confocal microscopy of pigmented basal cell carcinoma. J Am Acad Dermatol. 2006;54:638-43.

18. Shah KN. The risk of melanoma and neurocutaneous melanosis associated with congenital melanocytic nevi. Semin Cutan Med Surg. 2010;29:159-64.

19. Subbiah V, Wolff JE. Rapid response to therapy of neurocutaneous melanosis with leptomeningeal melanoma. Pediatr Blood Cancer. 2010;54:180-1.

20. • Phadke PA, Rakheja D, Le LP, et al. Proliferative nodules arising within congenital melanocytic nevi: a histologic, immunohistochemical, and molecular analyses of 43 cases. Am J Surg Pathol. 2011;35:656-69. This study suggested histological criteria and immunohistochemistry levels of Ki-67 and PHH3 were the strongest parameters to distinguish between atypical and benign proliferative nodules.

21. August PJ, Ferguson JE, Madan V. A study of the efficacy of carbon dioxide and pigment-specific lasers in the treatment of medium-sized congenital melanocytic naevi. Br J Dermatol. 2011;164:1037-42.
22. Funayama E, Sasaki S, Furukawa H, et al. Effectiveness of combined pulsed dye and Qs-ruby laser treatment for large to giant congenital melanocytic naevi. Br J Dermatol. 2012 [Epub ahead of print].

23. Tsao H, Bevona C, Goggins W, Quinn T. The transformation rate of moles (melanocytic nevi) into cutaneous melanoma: a population-based estimate. Arch Dermatol. 2003;139:282-8.

24. Gandini S, Sera F, Cattaruzza MS, et al. Meta-analysis of risk factors for cutaneous melanoma: I. Common and atypical naevi. Eur J Cancer. 2005;41:28-44.

25. Luther $\mathrm{H}$, Altmeyer $\mathrm{P}$, Garbe $\mathrm{C}$, et al. Increase of melanocytic nevus counts in children during 5 years of follow-up and analysis of associated factors. Arch Dermatol. 1996;132:1473-8.

26. - Zalaudek I, Schmid K, Marghoob AA, et al. Frequency of dermoscopic nevus subtypes by age and body site: a crosssectional study. Arch Dermatol. 2011;147:663-70. This study showed that a globular-pattern was the most prevalent pattern among acquired melanocytic nevi (AMN) on the upper trunk in children/adolescents and a reticular pattern was the most common among AMN after the second decade of life on the shoulders and middle back.

27. Scope A, Marghoob AA, Dusza SW, et al. Dermoscopic patterns of naevi in fifth grade children of the Framingham school system. Br J Dermatol. 2008;158:1041-9.

28. Zalaudek I, Grinschgl S, Argenziano G, et al. Age-related prevalence of dermoscopy patterns in acquired melanocytic naevi. Br J Dermatol. 2006;154:299-304.

29. Mahe E, Beauchet A, de Paula Correa M, et al. Outdoor sports and risk of ultraviolet radiation-related skin lesions in children: evaluation of risks and prevention. Br J Dermatol. 2011;165:360-7.

30. Pollock PM, Harper UL, Hansen KS, et al. High frequency of BRAF mutations in nevi. Nat Genet. 2003;33:19-20.

31. Albino AP, Nanus DM, Mentle IR, et al. Analysis of ras oncogenes in malignant melanoma and precursor lesions: correlation of point mutations with differentiation phenotype. Oncogene. 1989;4:1363-74.

32. Saldanha G, Purnell D, Fletcher A, et al. High BRAF mutation frequency does not characterize all melanocytic tumor types. Int $\mathrm{J}$ Cancer. 2004;111:705-10.

33. Clark Jr WH, Reimer RR, Greene M, et al. Origin of familial malignant melanomas from heritable melanocytic lesions. 'The B-K mole syndrome'. Arch Dermatol. 1978;114:732-8.

34. Greene MH, Reimer RR, Clark Jr WH, Mastrangelo MJ. Precursor lesions in familial melanoma. Semin Oncol. 1978;5:85-7.

35. Reimer RR, Clark Jr WH, Greene MH, et al. Precursor lesions in familial melanoma. A new genetic preneoplastic syndrome. JAMA. 1978;239:744-6.

36. •- Duffy K, Grossman D. The dysplastic nevus: From historical perspective to management in the modern era: Part I. Historical, histologic, and clinical aspects. J Am Acad Dermatol. 2012;67:1 e1-16. This article provides a comprehensive literature review of the historical, histologic, and clinical aspects of dysplastic nevi.

37. •• Duffy K, Grossman D. The dysplastic nevus: From historical perspective to management in the modern era: Part II. Molecular aspects and clinical management. J Am Acad Dermatol. 2012;67:19 e1-19. This article provides a comprehensive literature review of the molecular aspects and clinical management of dysplastic nevi.

38. Haley JC, Hood AF, Chuang TY, Rasmussen J. The frequency of histologically dysplastic nevi in 199 pediatric patients. Pediatr Dermatol. 2000;17:266-9.

39. Novakovic B, Clark Jr WH, Fears TR, et al. Melanocytic nevi, dysplastic nevi, and malignant melanoma in children from melanoma-prone families. J Am Acad Dermatol. 1995;33:631-6.

40. Foulkes WD, Flanders TY, Pollock PM, Hayward NK. The CDKN2A (p16) gene and human cancer. Mol Med. 1997;3:5-20.

41. Tcheung WJ, Marcello JE, Puri PK, et al. Evaluation of 39 cases of pediatric cutaneous head and neck melanoma. J Am Acad Dermatol. 2011;65:e37-42. 
42. - Tcheung WJ, Bellet JS, Prose NS, et al. Clinical and dermoscopic features of 88 scalp naevi in 39 children. Br J Dermatol. 2011;165:137-43. This study showed the most common dermatoscopic pattern of scalp nevi was globular and complex/reticularglobular pattern. Perifollicular hypopigmentation with resultant clinically scalloped borders or variegation in pigmentation also was a hallmark feature.

43. Moore MW, Gasparini R. FISH as an effective diagnostic tool for the management of challenging melanocytic lesions. Diagn Pathol. 2011;6:76.

44. Debarbieux S, Depaepe L, Poulalhon N, et al. Reflectance confocal microscopy accurately discriminates between benign and malignant melanocytic lesions exhibiting a 'dermoscopic island'. J Eur Acad Dermatol Venereol. 2012 [Epub ahead of print].

45. Helmbold P, Richter AM, Walesch S, et al. RASSF10 promoter hypermethylation is frequent in malignant melanoma of the skin but uncommon in nevus cell nevi. J Invest Dermatol. 2012;132:687-94.

46. Longo C, Rito C, Beretti F, et al. De novo melanoma and melanoma arising from pre-existing nevus: in vivo morphologic differences as evaluated by confocal microscopy. J Am Acad Dermatol. 2011;65:604-14.

47. Tom WL, Hsu JW, Eichenfield LF, Friedlander SF. Pediatric "STUMP" lesions: evaluation and management of difficult atypical Spitzoid lesions in children. J Am Acad Dermatol. 2011;64:559-72.

48. •• Luo S, Sepehr A, Tsao H. Spitz nevi and other Spitzoid lesions part I. Background and diagnoses. J Am Acad Dermatol. 2011;65:107384. This article provides a comprehensive literature review of the background and diagnosis of Spitz nevi.

49. Requena C, Botella R, Nagore E, et al. Characteristics of spitzoid melanoma and clues for differential diagnosis with spitz nevus. Am J Dermatopathol. 2012 [Epub ahead of print].

50. Kerl K, Kempf W, Kamarashev J, et al. Constitutional intraepidermal ascent of melanocytes: a potential pitfall in the diagnosis of melanocytic lesions. Arch Dermatol. 2012;148:235-8.

51. Gammon B, Beilfuss B, Guitart J, Gerami P. Enhanced detection of spitzoid melanomas using fluorescence in situ hybridization with 9p21 as an adjunctive probe. Am J Surg Pathol. 2012;36:81-8.

52. Cesinaro AM, Schirosi L, Bettelli S, et al. Alterations of 9p21 analysed by FISH and MLPA distinguish atypical spitzoid melanocytic tumours from conventional Spitz's nevi but do not predict their biological behaviour. Histopathology. 2010;57:515-27.

53. Martin V, Banfi S, Bordoni A, et al. Presence of cytogenetic abnormalities in Spitz naevi: a diagnostic challenge for fluorescence in-situ hybridization analysis. Histopathology. 2012;60:336-46.

54. Nardone B, Martini M, Busam K, et al. Integrating clinical/dermatoscopic findings and fluorescence in situ hybridization in diagnosing melanocytic neoplasms with less than definitive histopathologic features. J Am Acad Dermatol. 2012;66:917-22.

55. Al Dhaybi R, Agoumi M, Gagne I, et al. p16 expression: a marker of differentiation between childhood malignant melanomas and Spitz nevi. J Am Acad Dermatol. 2011;65:357-63.

56. Lazova R, Seeley EH, Keenan M, et al. Imaging mass spectrometry: a new and promising method to differentiate Spitz nevi from Spitzoid malignant melanomas. Am J Dermatopathol. 2012;34:82-90.

57. Nagaoka T, Nakamura A, Okutani H, et al. A possible melanoma discrimination index based on hyperspectral data: a pilot study. Skin Res Technol. 2011 [Epub ahead of print].

58. - Sepehr A, Chao E, Trefrey B, et al. Long-term outcome of Spitztype melanocytic tumors. Arch Dermatol. 2011;147:1173-9. This study showed that atypical Spitz tumors are associated with an increased melanoma risk, minimal lethal potential, and moderate risk of metastasis to the regional lymph node basin.

59. •• Luo S, Sepehr A, Tsao H. Spitz nevi and other Spitzoid lesions part II. Natural history and management. J Am Acad Dermatol. 2011;65:1087-92. This article provides a comprehensive literature review of the natural history and management of Spitz nevi.

60. Barnhill RL. The Spitzoid lesion: rethinking Spitz tumors, atypical variants, 'Spitzoid melanoma' and risk assessment. Mod Pathol. 2006;19 Suppl 2:S21-33.

61. Kim YJ, Han JH, Kang HY, et al. Androgen receptor overexpression in Becker nevus: histopathologic and immunohistochemical analysis. J Cutan Pathol. 2008;35:1121-6.

62. Patrizi A, Medri M, Raone B, et al. Clinical Characteristics of Becker's Nevus in Children: Report of 118 Cases from Italy. Pediatr Dermatol. 2012 [Epub ahead of print].

63. Ingordo V, Iannazzone SS, Cusano F, Naldi L. Dermoscopic features of congenital melanocytic nevus and Becker nevus in an adult male population: an analysis with a 10 -fold magnification. Dermatology. 2006;212:354-60.

64. Zeff RA, Freitag A, Grin CM, Grant-Kels JM. The immune response in halo nevi. J Am Acad Dermatol. 1997;37:620-4.

65. - van Geel N, Vandenhaute S, Speeckaert R, et al. Prognostic value and clinical significance of halo naevi regarding vitiligo. $\mathrm{Br} \mathrm{J}$ Dermatol. 2011;164:743-9. This study found the age of vitiligo onset among patients with halo nevi was significantly lower than patients without halo nevi.

66. Patrizi A, Bentivogli M, Raone B, et al. Association of halo nevus/i and vitiligo in childhood: a retrospective observational study. J Eur Acad Dermatol Venereol. 2012 [Epub ahead of print].

67. Lai C, Lockhart S, Mallory SB. Typical halo nevi in childhood: is a biopsy necessary? J Pediatr. 2001;138:283-4.

68. • Aouthmany M, Weinstein M, Zirwas MJ, Brodell RT. The natural history of halo nevi: A retrospective case series. J Am Acad Dermatol. 2012 [Epub ahead of print]. This study showed that a halo nevus typically persists for a decade or longer.

69. Diehl JW, Berk DR, Ney A, Bayliss SJ. Diffuse dermal melanocytosis: follow-up 30 years later with novel findings of eruptive blue nevi. Arch Dermatol. 2011;147:1339-40.

70. Singh S, Jain N, Khanna N, et al. Hairy nevus spilus: a case series. Pediatr Dermatol. 2012 [Epub ahead of print].

71. Corradin MT, Zattra E, Fiorentino R, et al. Nevus spilus and melanoma: case report and review of the literature. J Cutan Med Surg. 2010;14:85-9. 